\title{
Genetic Analysis of the rkp-3 Gene Region in Sinorhizobium meliloti 41: rkpY Directs Capsular Polysaccharide Synthesis to $\mathrm{K}_{\mathrm{R}} 5$ Antigen Production
}

\author{
Adrienn Pálvölgyi, ${ }^{1}$ Veronika Deák, ${ }^{1}$ Véréna Poinsot, ${ }^{2}$ Tibor Nagy, ${ }^{1}$ Enik Nagy, ${ }^{3}$ Ildikó Kerepesi, ${ }^{1}$ and \\ Péter Putnoky ${ }^{1}$ \\ ${ }^{1}$ Department of Genetics and Molecular Biology, University of Pécs, P.O. Box 266, H-7604 Pécs, Hungary; ${ }^{2}$ Laboratoire des \\ IMRCP, Université Paul Sabatier, F-31062 Toulouse, France; ${ }^{3}$ Institute of Genetics, Biological Research Center, Hungarian \\ Academy of Sciences, P.O. Box 521, H-6701 Szeged, Hungary
}

Submitted 17 May 2009. Accepted 17 June 2009.

\begin{abstract}
Rhizobial surface polysaccharides, including capsular polysaccharides (KPS), are involved in symbiotic infection. The rkp-3 locus of Sinorhizobium meliloti 41 is responsible for the production of pseudaminic acid, one of the components of the $K_{R} 5$ antigen, a strain-specific KPS. We have extended the sequence determination and genetic dissection of the $r k p-3$ region to clarify the structure and function of the $r k p Y$ gene and to identify additional $r k p$ genes. Except for $r k p Y$, no other genes were found where mutation affected the KPS structure and symbiosis. These mutants show a unique phenotype producing a low molecular weight polysaccharide (LMW PS). Creating double mutants, we have shown that biosynthesis genes of the $K_{\mathrm{R}} 5$ antigen except $r k p Z$ are not necessary for the production of this LMW PS. Polysaccharide analysis of genetically modified strains suggests that $r k p Y$ has pleiotropic effects on polysaccharide production. It directs KPS synthesis to the $K_{R} 5$ antigen and influences lipooligo 3-deoxy-d-manno-2 octulosonic acid (Kdo) production in $S$. meliloti 41. In addition, rkpY suppresses the lipo-oligoKdo production when it is introduced into $S$. meliloti 1021.
\end{abstract}

Gram-negative soil bacteria of Rhizobiaceae family belong to the $\alpha$-proteobacteria and contain the $\mathrm{N}_{2}$-fixing endosymbionts of legumes (Rhizobium, Azorhizobium, Bradyrhizobium, Sinorhizobium, and Mezorhizobium spp.) called rhizobia. Symbiotic plant-bacterium interaction begins with the induction of bacterial nodulation (nod) genes by specific plant flavonoids and with the initiation of a new plant organ, the nodule, in a host-specific manner by the product of Nod proteins. Invasion of the developing nodule by the bacteria is also a host-specific event where several additional bacterial genes are needed. Cell-surface polysaccharides of rhizobia were shown to be involved in several steps of the nodulation process, such as infection thread initiation and development, nodule invasion, and host specificity. However, unlike Nod factors, their

Corresponding author: P. Putnoky; Telephone: +36 (72) 503600 ext.4409; Fax: +36 (72) 503 634; E-mail: putnoky@ttk.pte.hu

Nucleotide sequence data are available at the GenBank/EMBL/DDBJ databases under accession numbers AJ249130 and AM849044. specific functions have not been elucidated (Fraysse et al. 2003; Jones et al. 2007).

Sinorhizobium meliloti produces several types of surface polysaccharides, including exopolysaccharides (EPS I and EPS II), lipopolysaccharide (LPS), cyclic glucans, and capsular polysaccharides (KPS or K-antigen). All of these components may be involved in the invasion process because mutant bacteria unable to produce one of these polysaccharides and to propagate within the infection thread were reported by several laboratories (Fraysse et al. 2003; Jones et al. 2007). S. meliloti 41 produces a strain-specific KPS, the $K_{R} 5$ antigen, which functionally compensates for the EPS I (succinoglycan) during the infection process, as was shown by using an EPS-deficient exoB mutant, AK631 (Putnoky et al. 1990; Petrovics et al. 1993). In an EPS-defective genetic background, the $K_{R} 5$ antigen of $S$. meliloti 41 is essential for the normal development of the infection threads and for the invasion of host plants. $\mathrm{Mu}-$ tants lacking the $K_{R} 5$ antigen produced aborted infection threads and failed to develop nitrogen-fixing symbiosis. Recently, the structure and genetics of this antigen were mainly elucidated. It is composed of low and high molecular weight (LMW and HMW) polysaccharides containing glucuronic acid (GlcA) and pseudaminic acid (Pse) disaccharide subunits which are decorated by butyryl and acetyl residues. This structure is analogous to the group II K antigens from Escherichia coli (Reuhs et al. 1993, 1998). A similar KPS structure was reported recently in Rhizobium sp. strain NGR234 (Le Quere et al. 2006) that also influences the development of symbiosis.

In $S$. meliloti 41 , three $r k p$ gene clusters ( $r k p-1, r k p-2$, and $r k p-3)$ involved in the $\mathrm{K}_{\mathrm{R}} 5$ antigen biosynthesis were identified (Putnoky et al. 1990; Kereszt et al. 1998; Kiss et al. 2001). Almost all of the rkp mutants show the same phenotypes. They are not able to i) produce wild-type KPS as the biochemical and immunological analysis indicated, ii) invade host plants in AK631 background, and iii) adsorb strain-specific bacteriophage 16-3 (Petrovics et al. 1993; Kereszt et al. 1998; Kiss et al. 1998).

The $r k p-1$ region harbors genes ( $r k p A$ to $r k p F)$ responsible for the synthesis of a specific lipid carrier for KPS biosynthesis, and genes ( $r k p G$ to $r k p J)$ involved in the modification and transfer of the carrier's lipophilic molecules (Petrovics et al. 1993; Kiss et al. 1997). Among these genes, $r k p F$ encoding an acyl carrier protein was studied more in detail (Epple et al. 1998). Interestingly, the $r k p A$ to $r k p F$ genes have been fused 
into a single large open reading frame (ORF) in strain $S$. meliloti 1021 (accession number AL591784) and in S. fredii HH103. In these strains, an additional $r k p$ gene encoding a putative export protein $(r k p U)$ was identified upstream of $r k p A$ (Parada et al. 2006). The $r k p U$ sequence of $S$. meliloti 41 was also determined (accession number AF247710) but its exact position remained unknown.

The $r k p-2$ region carries two genes (lpsL and $r k p K$ ), both necessary for wild-type LPS production. Moreover, $r k p K$ was shown to be required for the synthesis of a $K_{R} 5$ antigen precursor (UDP-GlcA) by encoding a UDP-glucose dehydrogenase (Kereszt et al. 1998).

In contrast to the above chromosomal regions, the $r k p-3$ region lies on the pRme41c megaplasmid $(\mathrm{pSymB})$ and contains several polysaccharide biosynthesis genes localized by genetic and DNA sequence analysis (Kereszt et al. 1998; Kiss et al. 2001). The rkpLMNOPQ gene cluster presumably performs the synthesis of the Pse component of the $\mathrm{K}_{\mathrm{R}} 5$ antigen. Three additional genes ( $r k p R, r k p S$, and $r k p T)$ encode for putative products with a high degree of similarity to polysaccharide transport proteins, but have no effect on either KPS biosynthesis or symbiosis. The right part of the published sequence (Kiss et al. 2001) encodes for RkpZ, which is homologous to polysaccharide chain-length-determinant proteins. The rkpZ insertional mutants show a size range modification of KPS (Reuhs et al. 1995). Outside of the sequenced region, approximately $6 \mathrm{~kb}$ downstream from $r k p Z$, an additional gene, $r k p Y$, was identified by Tn 5 insertions. These mutations affected the $\mathrm{K}_{\mathrm{R}} 5$ antigen production and resulted in invasion-defective and phage-16-3-resistant bacteria. A unique polysaccharide pattern was characteristic for these mutant strains (AT232 and AT233) (Kereszt et al. 1998). They produced only an LMW Alcian blue-staining material, supposed to represent an LMW derivative of the $\mathrm{K}_{\mathrm{R}} 5$ antigen.

In this study, we completed the sequence determination and genetic analysis of the $r k p-3$ region and showed by genetic experiments and polysaccharide analysis that $r k p Y$ mutants probably synthesize oligo 3-deoxy-d-manno-2 octulosonic acid (Kdo) but fail to produce $K_{R} 5$ antigen.

\section{RESULTS}

Nucleotide sequence of the $r k p Y$ region.

In order to clarify the structure of the $r k p Y$ gene and the existence of additional coding frames, we have determined the DNA sequence of a 6-kb Bam HI fragment (AJ249130). One large ORF encoding a 1,024-amino-acid (aa)-long putative protein $(116 \mathrm{kDa})$ was found in this region. All three Tn5 insertions (Kereszt et al. 1998; Kiss et al. 2001) that destroyed $\mathrm{K}_{\mathrm{R}} 5$ antigen production in this region were localized within this ORF by cloning and sequence analysis. Our results proved that this ORF represents the $r k p Y$ gene (Fig. 1A and B). Computer analysis of the putative RkpY protein highlighted no similarity to other protein sequences, and no characterized motifs or other functional domains were detected. The only structural characteristics of RkpY were one or two putative transmembrane domains. These data suggest that the $\operatorname{rkp} Y$ gene encodes for a novel type of protein.

Using additional subclones (data not shown), we have determined 12,289 nucleotide sequences in total (AM849044) expanding the DNA sequence of the $r k p-3$ region published earlier (Kiss et al. 2001). An ORF map of the extended $r k p-3$ region is shown in Figure 1A. In the newly sequenced part, eight ORF were found. In addition to $r k p Y$, a homologue of rkpT ( $r k p T 2$ ) represents a possible polysaccharide biosynthesis gene. The predicted product of this gene is an ABC-2 transporter permease, possibly involved in a cell surface polysaccharide export. It shares the highest identity $(58 \%)$ with RkpT1 of S. meliloti 1021. However, a comparison of the related protein sequences showed that the putative RkpT2 protein of $S$. meliloti 41 is more related to RkpT1 sequences from S. meliloti 1021, S. medicae WSM419, and Rhizobium sp. strain NGR234 than to the RkpT2 proteins from these species. The transmembrane prediction of RkpT2 showed five

A

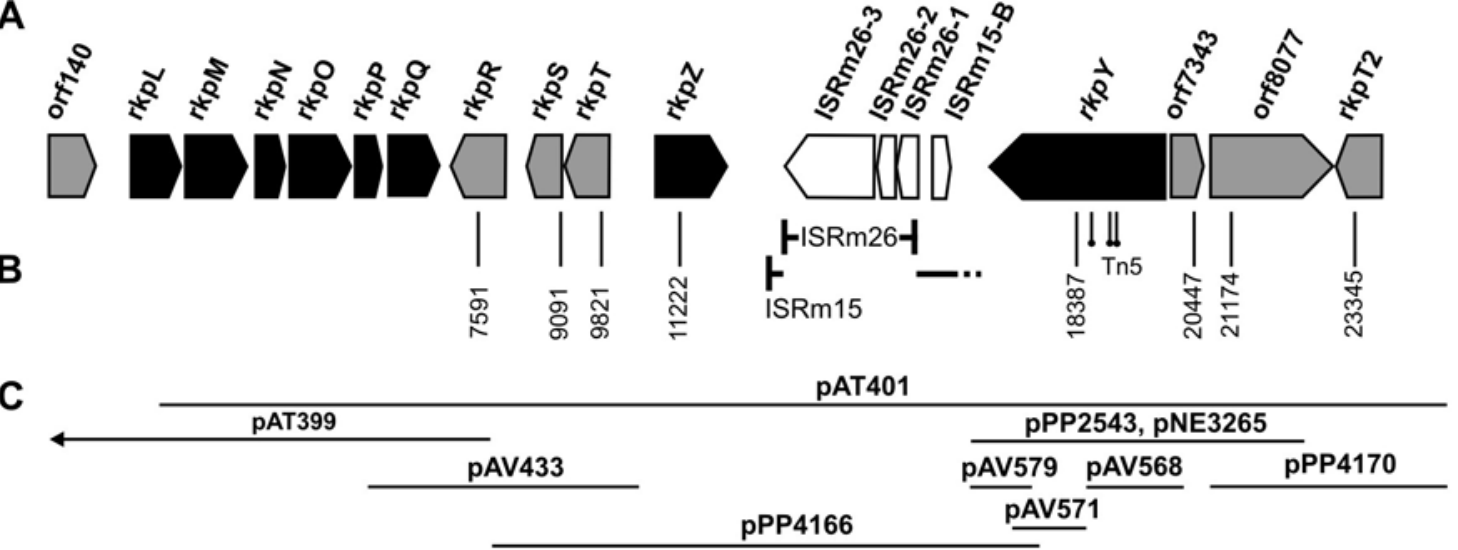

D

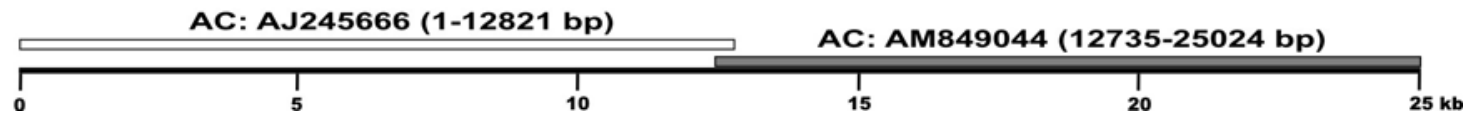

Fig. 1. Genetic map of the extended rkp-3 region in Sinorhizobium meliloti 41. A, Open reading frames (ORF) in the rkp-3 region. Black arrows represent genes involved in the biosynthesis of K-antigen; gray arrows show ORF encoding hypothetical proteins with unknown function; white arrows represent ORF encoding proteins with homology to those encoded in insertion elements ISRm 26 and ISRm15. B, Positions of the natural and generated insertion sequences in the region. A complete copy of ISRm26 disrupts ORF ISRm15-A. Full-length ORF ISRm15-B and a truncated copy of ISRm15-C sequences lay downstream from the ISRm26 insertion. Vertical bars with knobs represent the positions of the generated Tn5(66), Tn5(232), and Tn5(35) insertions. Simple vertical bars represent entranceposon insertions isolated in this study. Numbers show the exact coordinates of the insertions according to the unified $r k p$-3 sequence. C, Full lines represent inserts of different cosmid or plasmid derivatives used in this work. pAT399 and pAT401 are cosmid derivatives (Kiss et al. 2001); pBluescriptII SK(+) derivatives: NcoI fragment (pAV433), BamHI fragment (pPP2543), EcoRI fragments (pPP4166, pPP4170); pBBR1-MCS2 derivatives: BamHI fragment (pNE3265), SalI fragments (pAV568, pAV571), pBBR1-MCS5 derivative: BamHI-PstI fragment (pAV579). D, Range of sequences in the database records under the given accession numbers. The two records have 86 bp of overlap. 
to seven possible domains similar to that of Rkp1 of $S$. meliloti 41 .

The putative product of orf7343 (Fig. 1A) possesses a methyltransferase_11 domain (Pfam entry PF08241) and shares $72 \%$ identity with the predicted product of rkp3_012 from Rhizobium sp. strain NGR234 (ABD15245). The product of another putative gene, orf8077, harbors an ATP-binding region signature of protein kinases (ProSite entry PS00107) but has no homologues in the databases.

One complete insertion element (ISRm26) and a degraded copy of ISRm15 were identified upstream of the $r k p Y$ gene (Fig. 1B). ORF ISRm26-1, ISRm26-2, and ISRm26-3 and the 7-bp inverted repeat ends represent a complete copy of ISRm26. The 9-bp direct repeats of the target sequence were also identified. These findings suggest that this copy of ISRm26 is a functional one. Interestingly, it has been inserted into a truncated copy of ISRm 15 sequence containing only one complete coding region, ISRm15-B (protB). The ISRm15-A (protA) sequence was disrupted by the ISRm26, whereas the larger part of ISRm15-C (protC) sequence at the 3' end probably was removed earlier by a deletion event (Fig. 1B).

We performed an in silico search for the homologues of the $r k p-3$ genes. Several gene clusters similar to $r k p L M N O P Q$ were identified in different representatives of proteobacteria (data not shown). The putative product of these genes show homology to that of the pseB, pseC, pseF, pseG, pseH, and pseI genes of Campylobacter jejuni and Helicobacter pylori, where the biochemistry and genetics of Pse biosynthesis were studied in detail (Schirm et al. 2003; Guerry et al. 2006; Schoenhofen et al. 2006). Interestingly, as mentioned above, no homologues of $\operatorname{rkp} Y$ were found either in the vicinity of the putative pse gene clusters or anywhere in the complete genome sequences available in nucleotide databases. The only exception is Rhizobium sp. strain NGR234, where the presence of $r k p Y$ close to other $r k p$ genes were reported recently (Le Quere et al. 2006).

\section{Mutational analysis of the $r k p Y$ region.}

To investigate the possible function of the newly identified ORF (orf7343, orf8077, and rkpT2) in polysaccharide biosynthesis and symbiosis, a $\mathrm{Mu}$ entranceposon insertion mutagenesis was applied on appropriate subclones (Fig. 1C). Insertions car-

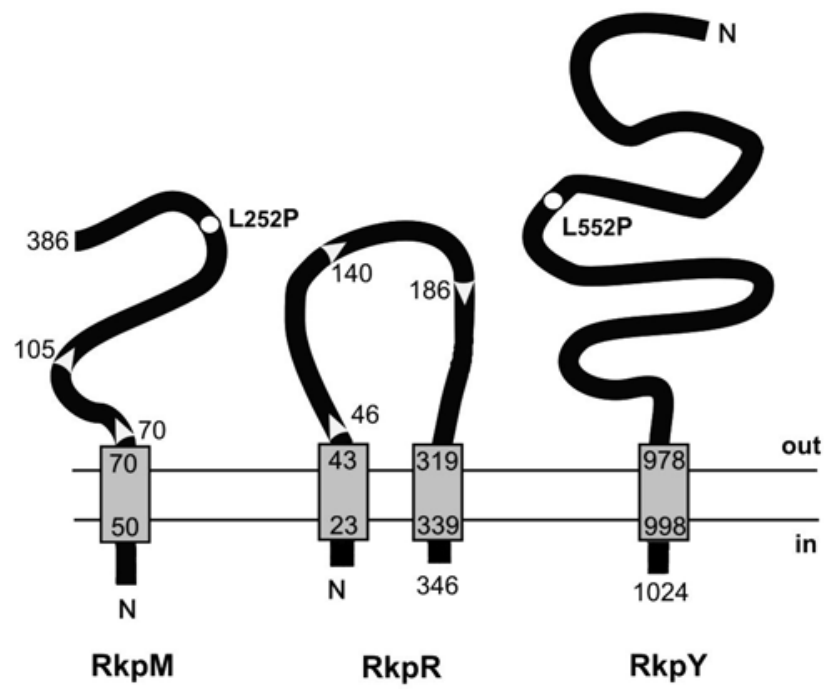

Fig. 2. Suggested structures of predicted transmembrane proteins RkpM, RkpR, and RkpY. Numbers represent i) the coordinates of the putative transmembrane domains (gray boxes) in amino acid residues, ii) the insertion of the PhoA fusions (at the arrows), iii) the receptor mutations (at the circles), and iv) the length of the predicted proteins (at the C-terminal ends). rying kanamycin resistance genes were physically mapped and sequenced. Representative insertions in the above ORF (Fig. 1B) were chosen to create homologous recombinants (as described below). Mutant bacteria were tested for their symbiotic ability, phage 16-3 resistance, and KPS or LPS production. No insertion resulting in any mutant phenotype was found, suggesting that orf7343, orf8077, and rkpT2 have no direct function in either polysaccharide production or symbiosis or the propagation of phage 16-3. Another reason for the lack of mutant phenotype could be functional redundancy elsewhere in the genome (Fig. 1A, rkpT1 and rkpT2).

In order to show whether the putative membrane proteins in the $r k p-3$ region harbor functional transmembrane domains, we performed $p h o A$ fusion experiments. Alkaline phosphatase (PhoA) is activated in the periplasmic space. The promoterless phoA gene of TnphoA transposon lacks its $5^{\prime}$ end coding for the signal peptide; therefore, most insertions result in no enzyme production. Translational fusion will only generate alkaline phosphatase activity if the protein fused to the truncated PhoA peptide contains one or more transmembrane domains that orient the C-terminal alkaline phosphatase part to the periplasm. Transposon TnphoA insertions were generated on cosmid pAT401 (Fig. 1C), and the target sequences of the insertions resulting in alkaline phosphatase activity in $S$. meliloti 41 were determined. Evaluating the sequence data, we could localize active translational fusions in the RkpM protein (after amino acid positions 70 and 105) (Fig. 2), the RkpR protein (after amino acid positions 46, 140, and 186) (Fig. 2), and the RkpT2 protein (after amino acid position 90).

However, all of our efforts to isolate active RkpY-PhoA fusions failed, although the $\operatorname{rkp} Y$ gene is three times longer than any of the above coding regions. The reason for this negative result may be that RkpY has either no transmembrane domains at all or a single C-terminal domain that is not suitable to isolate PhoA fusions by the method applied (Fig. 2) (discussed below).

\section{RkpY plays a direct role in phage 16-3 infection.}

Recently, we have described a special rkpM missense mutation that blocked the infection of the wild-type 16-3 phage but which made the isolation of host range phage mutants possible (Putnoky et al. 2004). We could also identify one spontaneous mutation in the $\operatorname{rkp} Y$ gene that resulted in a similar phenotype (PP4073; discussed below). In order to delimit the position of the mutation, marker rescue experiments were carried out. We took advantage of our observation that phage $M 1$ of $S$. meliloti 1021 could infect the $r k p$ mutants of $S$. meliloti 41 (Putnoky et al. 1990). Therefore, we examined which subfragment of the $r k p Y$ gene generates phage $M 1$-resistant wild-type bacteria by homologous recombination. Of the three subclones (Fig. 1C; pAV568, pAV571, and pAV579), only pAV571, representing the middle part of the gene, increased the appearance of $M 1$ resistant recombinants, suggesting that this fragment covers the mutation. After amplifying this region from strain PP4073, one missense mutation, a CCG codon instead of CTG, was identified that resulted in the L552P substitution (Fig. 2). The fact that a missense mutation of $r k p Y$ ensures the isolation of host range phage mutants supports the idea that the RkpY protein is involved directly in bacterium-phage recognition.

\section{Second mutations in $r k p$ genes except $r k p Z$ do not affect the LMW polysaccharide production in $r k p Y$ mutants.}

To test whether the LMW polysaccharide (LMW PS) produced by the $r k p Y$ mutants is a derivative of the $\mathrm{K}_{\mathrm{R}} 5$ antigen, as we previously supposed, we mutated essential $r k p$ genes in an $r k p Y$ mutant background. For this purpose, an $r k p Y$ spontaneous mutant, NE3222, was identified (as described below). 
To introduce the second mutations, different clones carrying transposon $\operatorname{Tn} 5$ insertions in representative $r k p$ genes were applied (Table 1). Homologous recombinants were examined for KPS production by deoxycholate polyacrylamide gel electrophoresis (DOC-PAGE).

Interestingly, the second mutations, except in the $r k p Z$ gene, did not influence the LMW PS production of the $r k p Y$ mutant (Table 1; Fig. 3), suggesting that the $r k p$ genes which are otherwise essential for $\mathrm{K}_{\mathrm{R}} 5$ antigen production are not involved in the biosynthesis of the LMW PS. In contrast, the rkpZ/rkpY mutant strain (AV476) produced no detectable amount of LMW PS (Fig. 3F). Another double mutant (AV518) was also created using a spontaneous rkpZ mutant (PP4180) and an entranceposon mutation of the $r k p Y$ gene. Again, the DOCPAGE analysis showed no detectable LMW PS (data not shown), suggesting that the rkpZ gene influences the synthesis of this unknown polysaccharide.

\section{rkpY suppresses Kdo homopolymer production in $S$. meliloti 1021.}

In the genome sequence of $S$. meliloti 1021, we could not detect any $r k p Y$-related gene. To test how the presence of $r k p Y$ influences the polysaccharide production of this strain, plasmid pNE3265 was conjugated into the wild-type background. This plasmid carries the rkpY gene (Fig. 1C) and it was shown to complement $r k p Y$ mutations in S. meliloti 41 (discussed below). DOC-PAGE analysis (Fig. 3) shows that the polysaccharide pattern of $S$. meliloti $1021\left(r k p Y^{+}\right)$differs from that of the wild type. Although the LMW PS in strain 1021 representing lipooligoKdo is produced in an abundant amount (Fig. 3G), the

Table 1. Deoxycholate polyacrylamide gel electrophoresis (DOC-PAGE) phenotypes of the $r k p-3$ and $r k p Y-r k p$ double mutants

\begin{tabular}{|c|c|c|}
\hline Strain & Genotype & KPS $^{\mathbf{a}}$ \\
\hline AK631 & Background strain producing $K_{R} 5$ antigen (Petrovics et al. 1993) & $w t$ \\
\hline PP4073 & $r k p Y_{4073}$ spontaneous mutant of AK631 (rkpY L552P) & $r k p Y$ \\
\hline NE3222 & $r k p Y_{3222}$ spontaneous mutant of AK631 & $r k p Y$ \\
\hline PP4180 & $r k p Z_{4180}$ spontaneous mutant (Putnoky et al. 2004) & $r k p Z$ \\
\hline AV488 & AK631 rkpR::ET-KanR-3 (7591) & $w t$ \\
\hline AV486 & AK631 rkpS:: ET-KanR-3 (9091) & $w t$ \\
\hline AV461 & AK631 rkpT::ET-KanR-3 (9821) & $w t$ \\
\hline AV475 & AK631 rkpZ::ET-KanR-3 (11222) & $r k p Z$ \\
\hline AV458 & AK631 rkpY::ET-KanR-3 (18387) & $r k p Y$ \\
\hline AV493 & AK631 orf7343::ET-KanR-3 (20447) & $w t$ \\
\hline AV457 & AK631 orf8077::ET-KanR-3 (21174) & $w t$ \\
\hline AV490 & AK631 rkpT2::ET-KanR-3 (23345) & $w t$ \\
\hline PP4141 & $r k p Y_{3222}+r k p A:: \operatorname{Tn} 5(666)$ & $r k p Y$ \\
\hline PP4140 & $r k p Y_{3222}+r k p B:: \operatorname{Tn} 5(665)$ & $r k p Y$ \\
\hline PP4139 & $r k p Y_{3222}+r k p C:: \operatorname{Tn} 5(644)$ & $r k p Y$ \\
\hline PP4199 & $r k p Y_{3222}+r k p K:: \operatorname{Tn} 5(207)$ & $r k p Y$ \\
\hline PP4201 & $r k p Y_{3222}+r k p L:: \operatorname{Tn} 5(187)$ & $r k p Y$ \\
\hline PP4202 & $r k p Y_{3222}+r k p M:: \operatorname{Tn} 5(212)$ & $r k p Y$ \\
\hline PP4200 & $r k p Y_{3222}+r k p Q:: \operatorname{Tn} 5(124)$ & $r k p Y$ \\
\hline PP3300 & $r k p Y_{3222}+\mathrm{pBBR} 1-\mathrm{MCS} 2:: r k p Y^{+}$ & $w t$ \\
\hline AV463 & $r k p Y_{3222}+r k p R:: \mathrm{ET}-\mathrm{KanR}-3(7591)$ & $r k p Y$ \\
\hline AV477 & $r k p Y_{3222}+r k p S::$ ET-KanR-3(9091) & $r k p Y$ \\
\hline AV479 & $r k p Y_{3222}+r k p T:: E T-K a n R-3(9821)$ & $r k p Y$ \\
\hline AV476 & $r k p Y_{3222}+r k p Z:: E T-K a n R-3(11222)$ & no KPS \\
\hline AV494 & $r k p Y_{3222}+$ orf7343::ET-KanR-3(20447) & $r k p Y$ \\
\hline AV483 & $r k p Y_{3222}+$ orf8077::ET-KanR-3(21174) & $r k p Y$ \\
\hline AV491 & $r k p Y_{3222}+r k p T 2:: E T-K a n R-3(23345)$ & $r k p Y$ \\
\hline AV518 & $r k p Z_{4180}+r k p Y:: E T-K a n R-3(18387)$ & No KPS \\
\hline
\end{tabular}

${ }^{a}$ KPS = capsular polysaccharides; wt, wild-type KPS pattern on DOC-PAGE; $r k p Z$, KPS production characteristic for $r k p Z$ mutants; $r k p Y$, low molecular weight (LMW) KPS production characteristic for $r k p Y$ mutants; no KPS, neither wild-type KPS nor LMW KPS characteristic for $r k p Y$ mutants were produced.

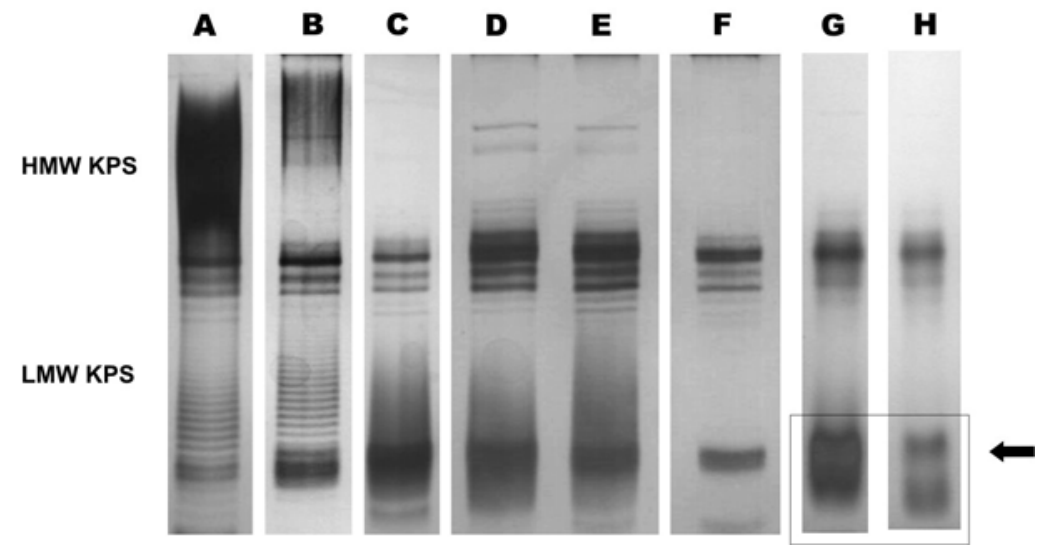

Fig. 3. Capsular polysaccharide (KPS) production by the rkp-3 mutants. A, Wild-type KPS from Sinorhizobium meliloti 41 (AK631); B, rkpZ phenotype characteristic for PP4180; C, $r k p Y$ phenotype, prototype $r k p Y:: \operatorname{Tn} 5(232)$; D and E, $r k p Y$ phenotype isolated from double mutants $r k p Y$-rkpA (PP4141) and rkpY-rkpL (PP4201), respectively; F, KPS pattern of the rkpY-rkpZ double mutant AV476; G, S. meliloti 1021 and $\mathbf{H}$, S. meliloti 1021 carrying the rkpY ${ }^{+}$ gene on pNE3265; arrow, predicted position of Kdo homopolymer (Sharypova et al. 2006). LMW and HMW = low and high molecular weight. 
introduction of $r k p Y$ resulted in a 10-fold reduction of this production (Fig. 3H). In the LPS pattern, no changes were detected (data not shown). Thus, the $\operatorname{rkp} Y$ gene from $S$. meliloti 41 is able to suppress the lipo-oligoKdo production in strain 1021.

\section{Structural characterization of polysaccharides.}

Three different media (YTA, TY, and YEM) were used for the propagation of bacteria to detect their possible influence on polysaccharide production. Strain AK631, the exoB mutant of $S$. meliloti 41, was conventionally used as background for the introduction of single and double mutations. As the crude extract analysis demonstrated, this parent strain produced an abundant amount of (GlcA-Pse)n oligo and polymers and a significant amount of lipo-oligoKdos (especially in TY medium) and glycerophosphate-substituted regular glucans (Fig. 4), as was reported recently (Chataigne et al. 2008). After mild hydrolysis of the AK631 polysaccharide crude extract, the KPS repetitive unit and Kdo were detected in significant quantities, as was the KPS lipid anchor $(\mathrm{m} / \mathrm{z} 622.48$ in the negative mode). In the $r k p Y_{3222}$ mutant, neither (GlcA-Pse)n nor lipo-oligoKdo could be detected. Only free oligoKdo and an important amount of glycerophosphorylated glucans could be observed (Fig. 4). The proportion and amount of these latter two components were dependent on the medium. After mild hydrolysis, no KPS lipid anchor could be detected. The rkpZ/rkpY double mutant produced only glycerophosphorylated glucans (Table 2).

When S. meliloti 1021 harbored the rkpY gene from strain 41 , it produced dramatically reduced lipo-oligoKdo compared with that of the parent strain. No lipid anchor (m/z 622.48) could be detected in the crude extract or after mild hydrolysis. Depending on the medium used for propagation, free oligoKdo (without anchor) could be detected in various amounts (Table 2). This result confirmed our observation in DOC-PAGE experiments that the presence of the $r k p Y$ gene in strain 1021 had a negative effect on the lipo-oligoKdo production.

\section{DISCUSSION}

Despite the growing body of evidence that KPS play an essential role in many plant-microbe interactions, there are relatively few data highlighting their importance in the invasion of nitrogen-fixing symbiotic nodules (Fraysse et al. 2003; Jones et al. 2007). Earlier, we identified several genes in the rkp-3 region of $S$. meliloti 41 (Kiss et al. 2001) that are respon-
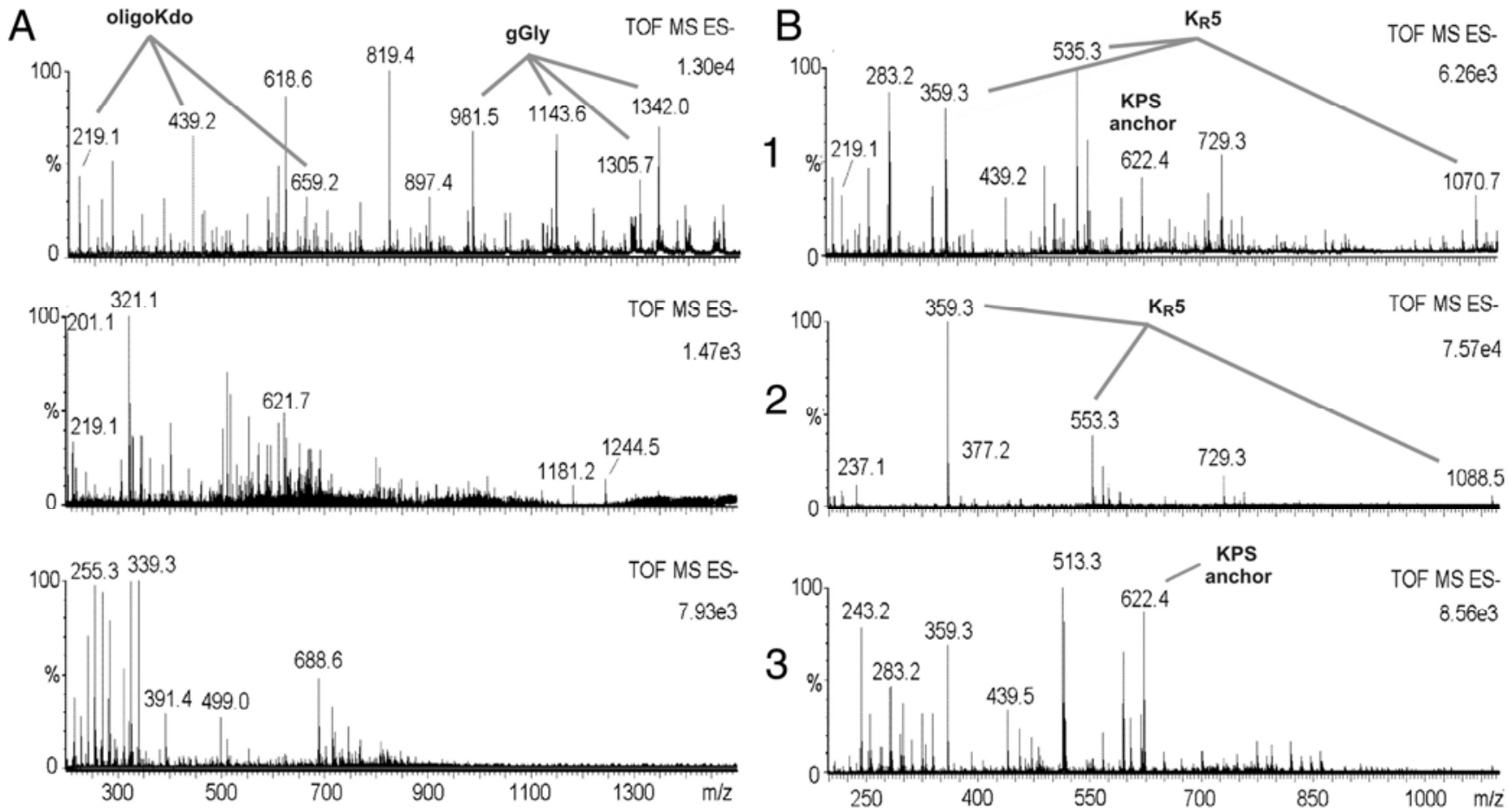

Fig. 4. Structural characterization of polysaccharides produced by the $r k p Y$ mutants. Electrospray ionization/quadrupole time-of-flight mass spectrometry (TOF MS ES) spectra of the 1, crude extract with in source energy; 2, saccharide fraction after mild hydrolysis; and $\mathbf{3}$, lipid fraction after mild hydrolysis for A, NE3222 and B, AK631. Ions at m/z 359, 535 (553), and 1,070 are characteristic for the high molecular weight capsular polysaccharides (KPS) (K $\mathrm{R}_{\mathrm{R}}$ ). $\mathrm{M} / \mathrm{z} 439$ and 659 are signatures for oligo 3-deoxy-d-manno-2 octulosonic acid (Kdo) and 622 for the lipo-oligoKdo anchor (KPS anchor). Ions at m/z 981, 1,143 , and 1,342 correspond to the glycerophosphorylated glycans (gGly).

Table 2. Chemical analysis of polysaccharide production of representative mutants ${ }^{\mathrm{a}}$

\begin{tabular}{|c|c|c|c|c|c|c|}
\hline Strain & Genotype & GlcA-Pse & Lipo oligoKdo & Free oligoKdo & Free anchor & $\beta$-Glucans \\
\hline AK631 & Sm41 exoB & ++++ & ++ & + & + & + \\
\hline NE3222 & $r k p Y_{3222}$ & - & - & $++^{\mathrm{b}}$ & - & $++^{\mathrm{b}}$ \\
\hline PP4180 & $r k p Z_{4180}$ & +++ & + & + & + & ++ \\
\hline AV476 & $r k p Y_{3222}$, rkpZ::ET-KanR-3 & - & - & $(-)$ & - & $+++^{\mathrm{b}}$ \\
\hline Sm1021 & Sinorhizobium meliloti 1021 wild type & - & ++++ & +++ & + & + \\
\hline AV4517 & S. meliloti $1021\left(r k p Y^{+}\right)$ & - & - & $t^{\mathrm{b}}$ & - & + \\
\hline
\end{tabular}

${ }^{a}$ GlcA, glucuronic acid; Pse, pseudaminic acid; KDO, 3-deoxy-d-manno-2 octulosonic acid; -, not detectable; +, less then 10\%; ++, approximately 40\%; +++ , approximately $70 \%$ of the maximum amount detected.

b Amount of polysaccharide depends on the medium used. Data shown were obtained using cells cultivated in YTA medium (Orosz et al. 1973) and harvested in the same growth phase. 
sible for the production of the $\mathrm{K}_{\mathrm{R}} 5$ antigen. This strain-specific KPS consists of disaccharide repeating units composed of GlcA and Pse (Reuhs et al. 1993, 1998). The rkpLMNOPQ gene cluster in the $r k p-3$ region was suggested to be involved in Pse biosynthesis, one of the component of the disaccharide subunit. In this work, we extended the DNA sequence determination of the $r k p-3$ region and presented the results of a genetic analysis, the aim of which was to clarify the possible function of the newly identified ORF.

On the basis of the mutant phenotypes, only one ORF, $r k p Y$, could be identified as an essential element of the $K_{R} 5$ antigen production, of the infection process in symbiosis, and of the infection of phage 16-3. Single-insertion mutations in orf7343, orf8077, and rkpT2 did not affect the above functions, suggesting that these putative genes are either not necessary or not essential for these events.

The putative RkpY protein does not possess any known motifs or domains except some transmembrane domains. The number of these predicted domains varies between 0 to 4 , depending on the program and the settings applied. To detect possible periplasmic segments of $\mathrm{RkpY}$ and other proteins encoded in the $r k p-3$ region, we isolated translational gene fusions using TnphoA (Manoil et al. 1990; Beckwith 2000). As expected, active fusions in $r k p M, r k p R$, and $r k p T 2$ could be successfully isolated. The three fusions localized in the middle of the $r k p R$ gene support one of the topology predictions, where an $\mathrm{N}$-terminal and a C-terminal transmembrane domain (residues 23 to 43 and 319 to 339) border a 275-aa-long extracellular domain (in which the fusions were localized) (Fig. 2).

A similar structure harboring a 616-aa-long extracellular domain $(1,848 \mathrm{bp}$ at DNA level) was also predicted for RkpY. However, we could not isolate any active fusion in the $r k p Y$ gene supporting this model. On the basis of this negative result, one may speculate that i) $\mathrm{RkpY}$ is not a membrane protein or, what seems to be more probable, ii) RkpY has only one transmembrane domain that was predicted at the very end of the protein (residues 978 to 998) (Fig. 2). Unfortunately, this latter topology makes the isolation of active gene fusions by TnphoA impossible, because the existence of an $\mathrm{N}$-terminal transmembrane domain that directs the $\mathrm{C}$-terminal fusion product to the periplasm is a prerequisite for the formation of active alkaline phosphatase (Manoil et al. 1990).

Other results presented here, however, indicate that RkpY is a membrane protein. We have identified a special missense mutation in allele $r k p Y L 552 \mathrm{P}$ that, in contrast to several other mutations in this gene, made the isolation of host range phage mutants possible. These host range phage mutants carry a missense mutation in gene $h$ (V. Deak, unpublished results), that probably encodes the tail fiber protein (Putnoky et al. 2004). These results suggest that residue L552 of RkpY is directly involved in the phage recognition or infection process. That is, $\mathrm{RkpY}$ functions as a membrane protein. It may possess only one C-terminal transmembrane domain that orient the 977-aalong N-terminal part outside (Fig. 2). Our recent data indicate that RkpY is associated with RkpM and RkpZ, because these proteins were also shown to be involved in phage 16-3 adsorption or infection process (Putnoky et al. 2004).

This study presents further evidence that RkpM is also a membrane protein. Because active phoA fusions were identified after position 70 and 105 residues, only the first of the two predicted transmembrane domains (residues 50 to 70 and 123 to 143) may exist that orients the C-terminal part outside (Fig. 2).

One of the basic questions is the function of the $r k p Y$ gene. The $r k p Y$ mutants produce an LMW, Alcian-blue-staining material that was supposed to be an LMW derivative of the $K_{R} 5$ antigen. Here, we have presented genetic evidence that this idea was wrong, because many essential biosynthesis genes of the $\mathrm{K}_{\mathrm{R}} 5$ antigen ( $r k p K, r k p L, r k p M$, and $r k p Q$ ) proved to be unnecessary for the production of this LMW PS. Polysaccharide analysis of different mutant strains supported the results of the genetic experiments. No $\mathrm{K}_{\mathrm{R}} 5$ antigen, (GlcA-Pse)n, was detected in the polysaccharide samples of an $r k p Y$ mutant. Moreover, both the defect of $r k p Y$ in strain 41 and the presence of the wild-type gene in strain 1021 disturbed the synthesis of lipo-oligoKdo, suggesting a possible role for RkpY in the lipidation process.

Previous studies indicated that $r k p Z 1$ mutation in strain 1021 destroyed the Kdo homopolimer production (Sharypova et al. 2006). In $r k p Z / r k p Y$ double mutants of strain 41, neither lipo-oligoKdo nor free oligoKdo were detected. This result suggests that the LMW PS visible in the DOC-PAGE of the $r k p Y$ mutants (Fig. 3C, D, and E) is probably oligoKdo.

Results presented here show that the $r k p Y$ gene is involved in the $K_{R} 5$ antigen as well as in the lipo-oligoKdo production and that it influences the amount of $\beta$-glucans presenting a pleiotropic effect on the polysaccharide production of the cells. To determine its exact function, further studies will be performed.

Two recent works have underlined the importance of KPS in symbiosis. A complete $r k p-3$ gene set except $r k p P$ was described in Rhizobium sp. strain NGR234. These genes are also necessary for the biosynthesis of a K-antigen, consisting of disaccharide repeating units of GlcA and Pse. It has a very similar structure to that of the $\mathrm{K}_{\mathrm{R}} 5$ antigen of $S$. meliloti 41 (Le Quere et al. 2006). Deletion mutant NGR $\triangle r k p M N O$ shows a reduced nodulation efficiency in different host plants, although the mutant bacteria could establish nitrogen-fixing symbiosis. The presence of an $r k p Y$ homologue sequence was also reported but its importance in KPS production and symbiosis has not yet been investigated.

Another rhizobia, S. fredii HH103, produces a Pse homopolymer (Gil-Serrano et al. 1999) that is essential for symbiosis, as was shown by $r k p-1$ region mutants (Parada et al. 2006). Although the presence of the $\operatorname{rkpLMNOPQ}$ gene cluster has not been reported, one may predict that a homologue gene cluster should be present in $S$. fredii $\mathrm{HH} 103$, similar to those bacteria that produce Pse.

A Pse biosynthesis pathway and the corresponding pse genes have been studied more in detail in Campilobacter and Helicobacter spp. (Schirm et al. 2003; Guerry et al. 2006). Interestingly, in these bacteria, the pse genes do not form one cluster although they located close to each other in the same chromosomal region. In rhizobia and in several other bacteria (as we found in the in silico analysis), the corresponding genes ( $r k p L M N O[P] Q)$ form one cluster. Interestingly, $r k p Y$ is not a fundamental element of these clusters. It was identified only in S. meliloti 41 and in Rhizobium sp. strain NGR234, that produces a structurally very similar Pse containing K-antigen. These strains provide good backgrounds to study how RkpY protein influences polysaccharide production.

\section{MATERIALS AND METHODS}

\section{Bacterial strains, bacteriophages, plasmids, and growth conditions.}

Rhizobium strains and plasmids used in this study are shown in Table 1 and Figure 1. E. coli strains XL1-Blue and DH5 $\alpha$ were used for transformation (Sambrook et al. 1989). For cloning and sequencing, plasmids pBluescriptII SK(+) (Stratagene, La Jolla, CA, U.S.A.), pUC19 (Yanisch-Perron et al. 1985), pBBR1-MCS2 and pBBR1-MCS5 (Kovach et al. 1995), and pPAG160 (Ganyu et al. 2005) were applied. Media, antibiotic concentrations, culture conditions for $E$. coli, and $S$. meliloti strains and phage 16-3 sensitivity tests have been described previously (Putnoky and Kondorosi 1986; Putnoky et al. 1990). 
Identification of spontaneous rkpY and host range phage 16-3 mutants.

We applied phage 16-3 as a selection tool for the isolation of $r k p$ mutants, as described earlier (Kereszt et al. 1998). Strains producing a KPS pattern in DOC-PAGE similar to that of $r k p Y:$ Tn5 mutants (Fig. 3C) were chosen for genetic complementation experiments. In all of these strains, $\mathrm{K}_{\mathrm{R}} 5$ antigen production and phage sensitivity were restored by the introduction of the wild-type allele of $r k p Y$ (Fig. 1C, pNE3265), supporting the idea that they have the defect in the $r k p Y$ gene. A representative mutant, NE3222, carrying $r k p Y_{3222}$ allele was chosen for the construction of the double mutants.

Mutant bacteria, resistant to wild-type phage 16-3 but sensitive to host range phage mutants, were isolated as described recently (Putnoky et al. 2004). An rkpY mutant strain, PP4073, was identified by the help of DOC-PAGE analysis and complementation experiments as described above. Three subclones of the rkpY gene (pAV568, pAV571, and pAV579) (Fig. 1C) were used in marker rescue experiments to delimit the mutation in PP4073. The nucleotide sequence of the mutant allele was established after isolating the appropriate DNA-region by PCR (discussed above).

\section{Transposon mutagenesis and homologous recombinants.}

Random transposon TnphoA insertions were generated on cosmid clone pAT401, as described previously (Putnoky et al. 1998). Insertions resulting in alkaline phosphatase activity in $S$. meliloti 41 were selected for sequence determination. The exact positions of the insertions were established by a phoAspecific reverse primer (GCAGTAATATCGCCCTGAGCAG) after subcloning the target sequence together with the kanamycin-resistant 5' end of TnphoA.

An in vitro transposon mutagenesis on plasmids pAV433, pPP2543, pPP4166, and pPP4170 (Fig. 1C) was carried out by the ET-KanR-3 Mu Entranceposon (TGS II kit; Finnzymes, Espoo, Finland) according to the instruction manual. Positions of the insertions were determined first by restriction mapping; then, some of them were chosen to establish the DNA sequence of the target region. Insertions localized in $r k p$ genes (Fig. 1B) were recloned with the target region into pPAG160 (Ganyu et al. 2005) for direct mutagenesis of the genomic sequence. Plasmid pPAG160 carries spectinomycin/streptomycin resistance $\left(\mathrm{Sp}^{\mathrm{r}} / \mathrm{Str}^{\mathrm{r}}\right)$, a pCU1 mob region, and a $\mathrm{pSC} 101$ replication origin (Thatte et al. 1985). It can be mobilized by pCU101 helper plasmid but it is unable to replicate in $S$. meliloti. Not only homologous recombination but also cointegrate formation and additional recombination events may result in kanamycinresistant derivatives; therefore, kanamycin- and spectinomycinsensitive transconjugants were tested for the presence of plasmid sequences by PCR using oligonucleotide primer pairs designed for the pSC101 region (GCTTGCGAGGGTGCTA CTTA, CGGTTACGAGATCCATTTGCT) and for the $\Omega$ fragment $\left(\mathrm{Sp}^{\mathrm{r}} / \mathrm{Str}^{\mathrm{r}}\right)$ (GCGCGATTTTGCCGGTTACT, GATGTTAC GCAGCAGGGCAGTC). In addition, locations of the insertions in the targeted genes were also confirmed by PCR. Recombinants harboring neither plasmid regions were chosen for further work. Representative Tn5 insertions in different $r k p$ genes (Table 1) were introduced on pLAFR1 cosmid clones into the AK631 genome via homologous recombination as described earlier (Kiss et al. 2001).

\section{DNA manipulations and sequence determination.}

Standard procedures, including DNA isolation, restriction enzyme digestion, agarose gel electrophoresis, DNA ligation, transformation, radioactive labeling, and Southern hybridization were performed using conventional methods (Sambrook et al. 1989) or as recommended by the suppliers. For sequence deter- mination, appropriate restriction fragments were subcloned into the vectors pUC19 or pBluescript II SK(+) and their nucleotide sequences were determined by the BigDyeTerminator kit on an Applied Biosystems 373A sequencer. Additional subsequences were obtained from Tn5-flanking regions of different insertional mutants using a $\mathrm{Tn} 5$-specific oligonucleotide primer (GCAAAA CGGGAAAGGTTCCG), and from ET-KanR-3 insertions using SeqE or SeqW primers (TGS II kit). The nucleotide sequence of the $r k p Y_{4073}$ allele was established after amplification with primer pair Y-Bam734U (AGCGAAATAGCGGGATGTTC) and Y-Bam2163L (GCCGGAATCCGTTTCTGA). For sequence determination, the above primers and two additional ones, YBam1375U (TTGCTAGCCGCTCCCGA) and Y-Bam1531L (CCTGGAGGGGCTGGTCTTT), were used.

DNA sequences were determined in at least two independent reactions at both strands. First, a 5,966-bp sequence of the $r k p Y$ region has been registered in the EMBL, GenBank, and DDBJ Nucleotide Sequence Databases under accession number AJ249130. Later, this sequence was extended in both directions, resulting in a 12,289-bp region and a new record (AM849044) that overlaps in 86 bp with the sequence record of the $r k p L-r k p Z$ region (AJ245666) published earlier (Kiss et al. 2001).

\section{Computer-assisted sequence analysis.}

PCGene software (designed by Amos Bairoch; Intelligenetics Corp., Mountain View, CA, U.S.A.) and the GCG program package (Devereux et al. 1984) were used for basic DNA sequence analysis and assembly. Amino acid homology searches were performed against the nonredundant database of the NCBI BLAST Network Service with the BlastP program. Protein family and domain predictions were performed with different proteomics tools from the ExPASy Molecular Biology Server. Transmembrane domains were calculated with the following programs: TopPred2 and DAS at the Stockholm Bioinformatics Center and SPLIT 4.0 at the University of Split. The codon preference program of the GCG software package was used to identify ORF showing codon usage similar to that of an average housekeeping $S$. meliloti gene as described previously (Kiss et al. 2001)

We performed a search for the homologues of the rkp-3 genes. First, BLASTP searches against the trEMBL database were carried out using the predicted Rkp protein sequences. When a homologue sequence was identified, the corresponding DNA region was examined for the presence of the whole gene cluster. Overall similarity to a given homologue was higher than $60 \%$.

\section{DOC-PAGE analysis of polysaccharides.}

Extraction of surface polysaccharides by a modified hot phenol-water method and DOC-PAGE analysis were carried out as described previously (Reuhs et al. 1993; Kiss et al. 1997). The phenol-water-extracted materials from bacteria were separated in 18\% DOC-PAGE and were visualized using Alcian blue silver staining. For KPS-specific analysis, the gels were stained without oxidation (Fig. 3); for LPS-specific staining, no Alcian blue was applied and sodium meta-periodate was used as oxidizer.

\section{Purification of LPS and KPS for chemical analysis.}

For chemical analyses, bacteria were cultured at $28^{\circ} \mathrm{C}$ in YTA (Orosz et al. 1973), TY (Beringer 1974), or YEM (Vincent 1970) medium. The cells were pelleted at early log phase, then washed by resuspending in deionized water followed by centrifugation. Polysaccharides were purified from representative mutants and were analyzed as described recently (Chataigne et al. 2008). Briefly, samples were extracted by the hot-phenol 
water method. The aqueous supernatant was dialyzed five times at 1:100 in water adjusted to $\mathrm{pH} 7.8$ with $\mathrm{NH}_{4} \mathrm{OH}$ (to minimize $\mathrm{Na}^{+}$and $\mathrm{Ca}^{2+}$ associations and optimize solubilization) using a $12,000-\mathrm{kDa}$ MWCO membrane. After the complete elimination of phenol traces, the water phases were made $10 \mathrm{mM}$ in $\mathrm{MgSO}_{4}$ and $50 \mathrm{mM}$ in Tris- $\mathrm{HCl}(\mathrm{pH} \mathrm{7.0)}$ and incubated with RNase and DNase for $48 \mathrm{~h}$ at $5^{\circ} \mathrm{C}$. Finally, proteinase $\mathrm{K}$ treatment was applied at $37^{\circ} \mathrm{C}$ for $4 \mathrm{~h}$. The sample were dialyzed again and freeze dried.

\section{Preparation of the saccharide and lipid part of the crude extracts.}

To release lipid anchor moieties from lipidated polysaccharides (lipidated oligoKdo and LPS), samples of the crude extracts $(1 \mathrm{mg} / \mathrm{ml})$ were treated with $1 \%$ acetic acid for $1 \mathrm{~h}$ at $100^{\circ} \mathrm{C}$. Then, the samples were centrifuged and the aqueous phases were extracted using chloroform in a 1:3 ( vol/vol) ratio to eliminate residual lipid anchor. The samples were evaporated at $40^{\circ} \mathrm{C}$ under a stream of filtered nitrogen.

\section{Electrospray ionization/quadrupole time-of-flight mass spectrometry analysis.}

Electrospray ionization/quadrupole time-of-flight (Q-tof) mass spectrometry (MS) analysis was performed in the negative-ion mode using a Q-tof Ultima Instrument (Waters, Milford, MA, U.S.A.).

Crude extract analysis. Samples were dissolved at a concentration of approximately $10 \mathrm{ng} / \mu \mathrm{l}$ in a 50:50:0.001 ( $\mathrm{vol} / \mathrm{vol} / \mathrm{vol})$ mixture of 2-propanol, water, and triethylamine and sprayed at a flow rate of $10 \mu \mathrm{l} / \mathrm{min}$. Capillary entrance voltage was set to $3.0 \mathrm{kV}$ and temperatures of source and dry gas were set to 100 and $120^{\circ} \mathrm{C}$, respectively. Settings were as follows: cone: $60 \mathrm{~V}$; Rf lens: $25 \mathrm{~V}$; and MS profile = m/z 150 (time 20\%), m/z 900 (time 60\%), and ramping $20 \%$.

Anchor analysis. Samples were dissolved at a concentration of approximately $50 \mathrm{ng} / \mu \mathrm{l}$ in a $\mathrm{CHCl}_{3} / \mathrm{MeOH} / \mathrm{H}_{2} \mathrm{O}$ (60:30:5) adjusted to $5 \mathrm{mM}$ in triethylamine. $\mathrm{MS}$ conditions are similar to the previous one, except that Cone voltage was $100 \mathrm{~V}$ and Rf lens was $70 \mathrm{~V}$.

The spectra, which showed several charge states for each component, were charge deconvoluted, and mass numbers were given refer to the monoisotopic molecular masses using the MaxEnt 3 software (MassLynx, Waters).

Mono and oligosaccharide analysis. Samples were dissolved at a concentration of $10 \mathrm{ng} / \mu \mathrm{l}$ in $\mathrm{MeOH} / \mathrm{H}_{2} \mathrm{O}(1: 1)$ adjusted to $5 \mathrm{mM}$ in triethylamine. MS conditions were the same as for the crude extracts.

\section{Plant tests.}

Assays for the symbiotic properties of the mutant Rhizobium meliloti strains were carried out with alfalfa (Medicago sativa L. Nagyszénási) plantlets, as previously described (Putnoky et al. 1988).

\section{ACKNOWLEDGMENTS}

This work was supported by grant OTKA T038377. We thank G. Jakab and $\mathrm{C}$. Fekete for advice in the preparation of the manuscript; and $\mathrm{M}$. Miklósvári, K. Garai, and J. Keidl for skillful technical assistance.

\section{LITERATURE CITED}

Beckwith, J. 2000. The all purpose gene fusion. Methods Enzymol. 326:3-7. Beringer, J. E. 1974. R-factor transfer of Rhizobium leguminosarum. J. Gen. Microbiol. 84:188-198.

Chataigne, G., Couderc, F., and Poinsot, V. 2008. Polysaccharides analysis of sinorhizobial capside by on-line anion exchange chromatography with pulsed amperometric detection and mass spectrometry coupling.
J. Chromatogr. A 1185:241-250.

Devereux, J., Haeberli, P., and Smithies, O. 1984. A comprehensive set of sequence analysis programs for the VAX. Nucleic Acids Res. 12:387395.

Epple, G., van der Drift, K. M., Thomas-Oates, J. E., and Geiger, O. 1998. Characterization of a novel acyl carrier protein, $\mathrm{RkpF}$, encoded by an operon involved in capsular polysaccharide biosynthesis in Sinorhizobium meliloti. J. Bacteriol. 180:4950-4954.

Fraysse, N., Couderc, F., and Poinsot, V. 2003. Surface polysaccharide involvement in establishing the Rhizobium-legume symbiosis. Eur. J. Biochem. 270:1365-1380.

Ganyu, A., Csiszovszki, Z., Ponyi, T., Kern, A., Buzas, Z., Orosz, L., and Papp, P. P. 2005. Identification of cohesive ends and genes encoding the terminase of phage 16-3. J. Bacteriol. 187:2526-2531.

Gil-Serrano, A. M., Rodriguez-Carvajal, M. A., Tejero-Mateo, P., Espartero, J. L., Menendez, M., Corzo, J., Ruiz-Sainz, J. E., and Buendia-Claveria, A. M. 1999. Structural determination of a 5-acetamido-3,5,7,9-tetradeoxy-7-(3-hydroxybutyramido)-L-glycero-L-manno-nonulosonic acidcontaining homopolysaccharide isolated from Sinorhizobium fredii HH103. Biochem. J. 342:527-535.

Guerry, P., Ewing, C. P., Schirm, M., Lorenzo, M., Kelly, J., Pattarini, D., Majam, G., Thibault, P., and Logan, S. 2006. Changes in flagellin glycosylation affect Campylobacter autoagglutination and virulence. Mol. Microbiol. 60:299-311.

Jones, K. M., Kobayashi, H., Davies, B. W., Taga, M. E., and Walker, G. C. 2007. How rhizobial symbionts invade plants: the SinorhizobiumMedicago model. Biochem. Biophys. Res. Commun. 5:619-633.

Kereszt, A., Kiss, E., Reuhs, B., Carlson, R. W., Kondorosi, A., and Putnoky, P. 1998. Novel rkp gene clusters of Sinorhizobium meliloti involved in capsular polysaccharide production and the invasion of the symbiotic nodule: $r k p K$ gene encodes for a UDP-glucose dehydrogenase. J. Bacteriol. 180:5426-5431.

Kiss, E., Reuhs, B., Kim, J., Kereszt, A., Petrovics, G., Putnoky, P., Dusha, I., Carlson, R. W., and Kondorosi, A. 1997. The rkpGHI and -J genes are involved in capsular polysaccharide production by Rhizobium meliloti. J. Bacteriol. 179:2132-2140.

Kiss, E., Mergaert, P., Olah, B., Kereszt, A., Staehelin, C., Davies, A. E., Downie, J. A., Kondorosi, A., and Kondorosi, E. 1998. Conservation of $n o l R$ in the Sinorhizobium and Rhizobium genera of the Rhizobiaceae family. Mol. Plant-Microbe Interact. 11:1186-1195.

Kiss, E., Kereszt, A., Barta, F., Stephens, S., Reuhs, B., L., Kondorosi, A., and Putnoky, P. 2001. The rkp-3 gene region of Sinorhizobium meliloti Rm41 contains strain-specific genes that determine K antigen structure. Mol. Plant-Microbe Interact. 14:1395-1403.

Kovach, M. E., Elzer, P. H., Hill, D. S., Robertson, G. T., Farris, M. A. Roop, R. M., and Peterson, K. M. 1995. Four new derivatives of the broad-host-range cloning vector pBBR1MCS, carrying different antibiotic-resistance cassettes. Gene 166:175-176.

Le Quere, A. J.-L., Deakin, W. J., Schmeisser, C., Carlson, R. W., Streit, W. R., Broughton, W. J., and Forsberg, L. S. 2006. Structural characterization of a K-antigen capsular polysaccharide essential for normal symbiotic infection in Rhizobium sp. NGR234. J. Biol. Chem. 281:2898128992.

Manoil, C., Mekalanos, J. J., and Beckwith, J. 1990 Alkaline phosphatase fusions: Sensors of subcellular location. J. Bacteriol. 172:515-518

Orosz, L., Svab, Z., Kondorosi, A., and Sik, T. 1973. Genetic studies on Rhizobio-phage 16-3. Genes and functions on the chromosome. Mol. Gen. Genet. 125:341-350.

Parada, M., Vinardell, J. M., Ollero, F. J., Hidalgo, A., Gutierrez, R., Buendia-Claveria, A. M., Lei, W., Margaret, I., Lopez-Baena, F. J., GilSerrano, A. M., Rodriguez-Carvajal, M. A., Moreno, J., and Ruiz-Sainz, J. E. 2006. Sinorhizobium fredii HH103 mutants affected in capsular polysaccharide (KPS) are impaired for nodulation with soybean and Cajanus cajan. Mol. Plant-Microbe Interact. 19:43-52.

Petrovics, G., Putnoky, P., Reuhs, B., Kim, J., Thorp, T. A., Noel, K. D., Carlson, R. W., and Kondorosi, A. 1993. The presence of a novel type of surface polysaccharide in Rhizobium meliloti requires a new fatty acid synthase-like gene cluster involved in symbiotic nodule development. Mol. Microbiol. 8:1083-1094.

Putnoky, P., and Kondorosi, A. 1986. Two gene clusters of Rhizobium meliloti code for early essential nodulation functions and a third influences nodulation efficiency. J. Bacteriol. 167:881-887.

Putnoky, P., Grosskopf, E., Ha, D. T. C., Kiss, G. B., and Kondorosi, A. 1988. Rhizobium fix genes mediate at least two communication steps in symbiotic nodule development. J. Cell Biol. 106:597-607.

Putnoky, P., Petrovics, G., Kereszt, A., Grosskopf, E., Ha, D. T. C., Banfalvi, Z., and Kondorosi, A. 1990. Rhizobium meliloti lipopolysaccharide and exopolysaccharide can have the same function in the plant-bacterium interaction. J. Bacteriol. 172:5450-5458.

Putnoky, P., Kereszt, A., Nakamura, T., Endre, G., Grosskopf, E., Kiss, P., 
and Kondorosi, A. 1998. The pha gene cluster of Rhizobium meliloti involved in $\mathrm{pH}$ adaptation and symbiosis encodes a novel type of K+efflux system. Mol. Microbiol. 28:1091-1101.

Putnoky, P., Deák, V., Békási, K., Pálvölgyi, A., Maász, A., Palágyi, Z., Hoffmann, G., and Kerepesi, I. 2004. H protein of bacteriophage 16-3 and RkpM protein of Sinorhizobium meliloti 41 are involved in phage adsorbtion. J. Bacteriol. 186:1591-1597.

Reuhs, B. L., Carlson, R. W., and Kim, J. S. 1993. Rhizobium fredii and Rhizobium meliloti produce 3-deoxy-D-manno-2- octulosonic acid-containing polysaccharides that are structurally analogous to group II K antigens (capsular polysaccharides) found in Escherichia coli. J. Bacteriol. 175:3570-3580.

Reuhs, B. L., Williams, M. N., Kim, J. S., Carlson, R. W., and Cote, F 1995. Suppression of the Fix- phenotype of Rhizobium meliloti exoB mutants by lps $Z$ is correlated to a modified expression of the $\mathrm{K}$ polysaccharide. J. Bacteriol. 177:4289-4296.

Reuhs, B. L., Geller, D. P., Kim, J. S., Fox, J. E., and Pueppke, S. G. 1998. Sinorhizobium fredii, and Sinorhizobium meliloti produce structurally conserved lipopolysaccharides and strain-specific K antigens. Appl. Environ. Microbiol. 64:4930-4938.

Sambrook, J., Fritsch, E. F., and Maniatis, T. 1989. Molecular Cloning: A Laboratory Manual, 2nd ed. Cold Spring Harbor Laboratory Press, Cold Spring Harbor, NY, U.S.A.

Schirm, M., Soo, E. C., Aubry, A. J., Austin, J., Thibault, P., and Logan, S. M. 2003. Structural, genetic and functional characterization of the flag- ellin glycosylation process in Helicobacter pylori. Mol. Microbiol. 48:1579-1592.

Schoenhofen, I. C., McNally, D. J., Brisson, J.-R., and Logan, S. M. 2006. Elucidation of the CMP-pseudaminic acid pathway in Helicobacter pylori: synthesis from UDP-N-acetylglucosamine by a single enzymatic reaction. Glycobiology 16:8C-14C.

Sharypova, L. A., Chataigné, G., Fraysse, N., Becker, A., and Poinsot, V. 2006. Overproduction and increased molecular weight account for the symbiotic activity of the rkpZ-modified K polysaccharide from Sinorhizobium meliloti Rm1021. Glycobiology 16:1181-1193.

Thatte, V., Bradley, D. E., and Iyer, V. N. 1985. N conjugative transfer system of plasmid pCU1. J. Bacteriol. 163:1229-1236.

Vincent, J. M., ed. 1970. A Manual for the Practical Study of Root-Nodule Bacteria. Blackwells, Oxford.

Yanisch-Perron, C., Viera, J., and Messing, J. 1985. Improved M13 phage cloning vectors and host strains: nucleotide sequence of the M13mp18 and pUC19 vectors. Gene 33:103-119.

\section{AUTHOR-RECOMMENDED INTERNET RESOURCES}

NCBI BLAST Network Service: www.ncbi.nlm.nih.gov/blast/Blast.cgi

ExPASy Molecular Biology Server: www.expasy.org

Stockholm Bioinformatics Center: www.sbc.su.se/services

University of Split: split.pmfst.hr/split/4 